\title{
On Geometric Complexity Theory: Multiplicity Obstructions Are Stronger Than Occurrence Obstructions
}

\author{
Julian Dörfler \\ Saarland University, Saarbrücken, Germany \\ s8judoer@stud.uni-saarland.de
}

\section{Christian Ikenmeyer}

Max Planck Institute for Software Systems, Saarbrücken, Germany

cikenmey@mpi-sws.org

\section{Greta Panova}

University of Southern Californa, Los Angeles, CA, USA

University of Pennsylvania, Philadelphia, PA, USA

gpanova@usc.edu

\begin{abstract}
Geometric Complexity Theory as initiated by Mulmuley and Sohoni in two papers (SIAM J Comput 2001, 2008) aims to separate algebraic complexity classes via representation theoretic multiplicities in coordinate rings of specific group varieties. We provide the first toy setting in which a separation can be achieved for a family of polynomials via these multiplicities.

Mulmuley and Sohoni's papers also conjecture that the vanishing behavior of multiplicities would be sufficient to separate complexity classes (so-called occurrence obstructions). The existence of such strong occurrence obstructions has been recently disproven in 2016 in two successive papers, Ikenmeyer-Panova (Adv. Math.) and Bürgisser-Ikenmeyer-Panova (J. AMS). This raises the question whether separating group varieties via representation theoretic multiplicities is stronger than separating them via occurrences. We provide first finite settings where a separation via multiplicities can be achieved, while the separation via occurrences is provably impossible. These settings are surprisingly simple and natural: We study the variety of products of homogeneous linear forms (the so-called Chow variety) and the variety of polynomials of bounded border Waring rank (i.e. a higher secant variety of the Veronese variety).
\end{abstract}

As a side result we prove a slight generalization of Hermite's reciprocity theorem, which proves Foulkes' conjecture for a new infinite family of cases.

2012 ACM Subject Classification Theory of computation $\rightarrow$ Algebraic complexity theory

Keywords and phrases Algebraic complexity theory, geometric complexity theory, Waring rank, plethysm coefficients, occurrence obstructions, multiplicity obstructions

Digital Object Identifier 10.4230/LIPIcs.ICALP.2019.51

Category Track A: Algorithms, Complexity and Games

Related Version A full version of the paper is available at https://arxiv.org/abs/1901.04576.

Funding Julian Dörfler: Partially supported by DFG grant IK 116/2-1.

Christian Ikenmeyer: Partially supported by DFG grant IK 116/2-1.

Greta Panova: Partially funded by the NSF.

Acknowledgements This work was done in part while CI and GP were visiting the Simons Institute for the Theory of Computing.

(c) (i) Julian Dörfler, Christian Ikenmeyer, and Greta Panova;

C. licensed under Creative Commons License CC-BY

46th International Colloquium on Automata, Languages, and Programming (ICALP 2019). Editors: Christel Baier, Ioannis Chatzigiannakis, Paola Flocchini, and Stefano Leonardi; Article No. 51; pp. 51:1-51:14

Leibniz International Proceedings in Informatics
LIPICS Schloss Dagstuhl - Leibniz-Zentrum für Informatik 


\section{Introduction}

In two landmark papers [22, 23] Mulmuley and Sohoni suggested the use of representation theoretic multiplicities to separate group varieties that correspond to complexity classes. The goal of this approach, which is called geometric complexity theory, is to achieve complexity lower bounds that lead to the separation of algebraic complexity classes such as VP and VNP (see [3] or [28] for the precise definitions, which will not be important in this paper). At the heart of the approach was the hope that so-called occurrence obstructions (see Section 2) would be sufficient to separate VP and VNP. In $[16,8]$ it was shown that occurrence obstructions are too weak to provide the necessary separation, at least for the group varieties that were originally proposed by Mulmuley and Sohoni. But representation theoretic multiplicities might still be able to separate VP and VNP when we look at the finer separation criterion via multiplicity obstructions (see also Section 2). Unfortunately, so far all known separations of group varieties via multiplicity obstructions could also in fact be obtained via occurrence obstructions, or at least there is no setting in which multiplicity obstructions are provably stronger than occurrence obstructions, see e.g. [6, 7]. Indeed, little is known about multiplicity obstructions in general, as the required multiplicities are often \#P-hard to compute, see e.g. $[25,5,2]$, which implies that a polynomial time algorithm for their computation can only exist if $\mathrm{P}=\mathrm{NP}$.

Scott Aaronson raised the question about the existence of a setting where multiplicity obstructions are provably more powerful than occurrence obstructions. In this paper we give the first example of such a situation in a finite setting, see Theorem 2.1 below.

Theorem 2.1 is not only about finite settings: For the first time multiplicity obstructions are used to separate families of polynomials, even though the separation is extremely modest. Prior work on obstructions focused on tensors instead of polynomials $([6,7])$.

As a side result we prove a slight generalization of Hermite's reciprocity theorem, which proves Foulkes' conjecture (see (1)) for a new infinite family of cases, see Theorem 3.4.

\section{Representation theoretic obstructions}

In this section we review how to separate group varieties via representation theoretic multiplicities. The setup is in complete analogy to the geometric complexity theory approach of Mulmuley and Sohoni. We then list our main result, see Theorem 2.1.

Consider the space $\mathbb{A}_{m}^{n}:=\mathbb{C}\left[x_{1}, \ldots, x_{m}\right]_{n}$ of complex homogeneous polynomials of degree $n$ in $m$ variables. Let $V:=\mathbb{A}_{m}^{1}$ be the space of homogeneous degree 1 polynomials. In this paper we compare two subvarieties of $\mathbb{A}_{m}^{n}$. The first is the so-called Chow variety

$$
\mathrm{Ch}_{m}^{n}:=\left\{\ell_{1} \cdots \ell_{n} \mid \ell_{i} \in V\right\} \subseteq \mathbb{A}_{m}^{n},
$$

which is the set of polynomials that can be written as a product of homogeneous linear forms, see e.g. $[20, \S 8.6]$. In algebraic complexity theory this set is known as the set of polynomials that have homogeneous depth-two algebraic circuits of the form $\Pi^{n} \Sigma$, i.e., circuits that consists of an $n$-ary top product gate of linear combinations of variables. The second variety is called a higher secant variety of the Veronese variety and can be written as

$$
\operatorname{Pow}_{m, k}^{n}:=\overline{\left\{\ell_{1}^{n}+\cdots+\ell_{k}^{n} \mid \ell_{i} \in V\right\}} \subseteq \mathbb{A}_{m}^{n},
$$

which is the closure of the set of all sums of $k$ powers of homogeneous linear forms. Note that from a general principle it follows that the Zariski closure equals the Euclidean closure in this case, see e.g. $[24, \S 2 . \mathrm{C}]$ where this is shown for every constructible set. The polynomials 
in Pow ${ }_{m, k}^{n}$ are exactly those that have border Waring rank at most $k$, see e.g. [20, §5.4]. In algebraic complexity theory this set is known as the set of polynomials that can be approximated arbitrarily closely by homogeneous depth-three powering circuits of the form $\Sigma^{k} \Lambda^{n} \Sigma$, i.e., a $k$-ary sum of $n$-th powers of linear combinations of variables.

$\mathbb{A}_{m}^{n}$ is generated as a vector space by the powers $v^{n}, v \in V$, see e.g. [20, Ex. 2.6.6.2]. Given two elements $g_{1}, g_{2} \in \mathrm{GL}_{m}:=\mathrm{GL}(V)$, and given $v \in V$, we clearly have $g_{1}\left(g_{2} v\right)=\left(g_{1} g_{2}\right) v$. Thus we say that $V$ admits a $\mathrm{GL}_{m}$-action. This natural action of $\mathrm{GL}_{m}$ on $V$ lifts canonically to $\mathbb{A}_{m}^{n}$ via $g\left(v^{n}\right):=(g v)^{n}, g \in \mathrm{GL}_{m}, v \in V$, and linear continuation. Both varieties $\mathrm{Ch}_{m}^{n}$ and Pow $_{m, k}^{n}$ are closed under this action, i.e., for $g \in \mathrm{GL}_{m}$ and $v \in \mathrm{Ch}_{m}^{n}$ we have $g v \in \mathrm{Ch}_{m}^{n}$, and analogously $v \in \operatorname{Pow}_{m, k}^{n}$ implies $g v \in \operatorname{Pow}_{m, k}^{n}$. A variety that is closed under the action of $\mathrm{GL}_{m}$ is called a $\mathrm{GL}_{m}$-variety.

Let $\mathbb{C}\left[\mathbb{A}_{m}^{n}\right]$ denote the coordinate ring of $\mathbb{A}_{m}^{n}$, i.e., the polynomial ring in $\operatorname{dim} \mathbb{A}_{m}^{n}=$ $\left(\begin{array}{c}n+m-1 \\ n\end{array}\right)$ many variables, where these variables are in 1:1 correspondence to the monomials in $\mathbb{A}_{m}^{n}$. The action of $\mathrm{GL}_{m}$ on $\mathbb{A}_{m}^{n}$ lifts to a linear action of $\mathrm{GL}_{m}$ on $\mathbb{C}\left[\mathbb{A}_{m}^{n}\right]$ via the canonical pullback as follows: $(g f)(h):=f\left(g^{-1} h\right), g \in \mathrm{GL}_{m}, f \in \mathbb{C}\left[\mathbb{A}_{m}^{n}\right], h \in \mathbb{A}_{m}^{n}$. Moreover, the action respects the natural grading of $\mathbb{C}\left[\mathbb{A}_{m}^{n}\right]$, so that each homogeneous degree $d$ part $\mathbb{C}\left[\mathbb{A}_{m}^{n}\right]_{d}$ is a finite dimensional vector space that is closed under the action of $\mathrm{GL}_{m}$.

Recall that a finite dimensional vector space $W$ that is closed under a linear action of $\mathrm{GL}_{m}$ is called a $\mathrm{GL}_{m}$-representation. This is equivalent to the existence of a group homomorphism $\varrho: \mathrm{GL}_{m} \rightarrow \mathrm{GL}(W)$. If we choose bases, then we can interpret $\mathrm{GL}_{m} \subseteq \mathbb{C}^{m \times m}$ and $\mathrm{GL}(W) \subseteq \mathbb{C}^{\operatorname{dim} W \times \operatorname{dim} W}$ and $\varrho$ is described by $(\operatorname{dim} W)^{2}$ many coordinate functions, which are functions in $m^{2}$ many variables. If these functions are polynomials, then we call $W$ a polynomial representation. Our main representation of interest, $\mathbb{C}\left[\mathbb{A}_{m}^{n}\right]_{d}$, is a polynomial representation. A linear subspace of $W$ that is closed under the action of $\mathrm{GL}_{m}$ is called a subrepresentation. Subrepresentations of polynomial representations are clearly polynomial representations again. For every $\mathrm{GL}_{m}$-representation $W$ we have that $W$ and 0 are two trivial subrepresentations. If $W$ has no other subrepresentations, then we call $W$ irreducible. A linear map $\varphi: W_{1} \rightarrow W_{2}$ between two $\mathrm{GL}_{m}$-representations is called equivariant if $g \varphi(f)=\varphi(g f)$ for all $f \in W_{1}, g \in \mathrm{GL}_{m}$. If there exists an equivariant vector space isomorphism from $W_{1}$ to $W_{2}$, then we say that $W_{1}$ and $W_{2}$ are isomorphic $\mathrm{GL}_{m}$-representations. An m-partition of $D$ is a nonincreasing list of $m$ nonnegative integers that sum up to $D$. Every irreducible polynomial $\mathrm{GL}_{m}$-representation has an associated isomorphism type, which is an $m$-partition, see e.g. [10, Ch. 8]. Two irreducible $\mathrm{GL}_{m}$-representations are isomorphic iff their isomorphism types coincide. We denote by $\{\lambda\}_{m}$ the irreducible $\mathrm{GL}_{m}$-representation corresponding to the $m$-partition $\lambda$. We write $\{\lambda\}=\{\lambda\}_{m}$ is $m$ is clear from the context.

The group $\mathrm{GL}_{m}$ is linearly reductive, which means that every $\mathrm{GL}_{m}$-representation $W$ decomposes into a direct sum of irreducible $\mathrm{GL}_{m}$-representations, see e.g. [18, AII.5, Satz 4]. The number of times an irreducible representation of type $\lambda$ occurs in the decomposition is called the multiplicity of $\lambda$ in $W$, written $\operatorname{mult}_{\lambda}(W)$. Even though this decomposition is usually not unique, the notation $\operatorname{mult}_{\lambda}(W)$ makes sense, because the multiplicities are independent of the actual decompositions.

The multiplicity $a_{\lambda}(d[n]):=\operatorname{mult}_{\lambda}\left(\mathbb{C}\left[\mathbb{A}_{m}^{n}\right]_{d}\right)$ is the infamous plethysm coefficient, which is the object of study in Foulkes' conjecture and also in Problem 9 in Stanley's famous list of open problems [30]. If we pad an $m$-partition $\lambda$ with $m^{\prime}-m$ many zeros to obtain the $m^{\prime}$-partitions $\lambda^{\prime}=\left(\lambda_{1}, \ldots, \lambda_{m}, 0, \ldots, 0\right)$, then $\operatorname{mult}_{\lambda}\left(\mathbb{C}\left[\mathbb{A}_{m}^{n}\right]_{d}\right)=\operatorname{mult}_{\lambda^{\prime}}\left(\mathbb{C}\left[\mathbb{A}_{m^{\prime}}^{n}\right]_{d}\right)$, see e.g. [14, Lem. 4.3.2]. For the sake of simplicity we identify $m$-partitions with $m^{\prime}$-partitions that arise from padding zeros. This justifies leaving out the parameter $m$ in the notation $a_{\lambda}(d[n])$ by assuming that $m$ is large enough. Foulkes' conjecture states that

Conjecture : $\quad a_{\lambda}(n[d]) \leq a_{\lambda}(d[n])$ for all $d \geq n$. 
Conjecture (1) is known to be true (moreover, equality holds: $a_{\lambda}(d[n])=a_{\lambda}(n[d])$ ) for all 2-partitions $\lambda$, which is often called Hermite reciprocity [13]. We make modest progress on this conjecture by proving it for many families of 3-partitions, see Corollary 4.4.

Let $Z$ be a $\mathrm{GL}_{m}$-variety, e.g., $Z=\mathrm{Ch}_{m}^{n}$ or $Z=\operatorname{Pow}_{m, k}^{n}$. Then the vanishing ideal $I(Z):=\left\{f \in \mathbb{C}\left[\mathbb{A}_{m}^{n}\right] \mid \forall h \in Z: f(h)=0\right\}$ is also closed under the action of $\mathrm{GL}_{m}$, which is easy to verify: If $f(h)=0$ for all $h \in Z$, then also $(g f)(h)=f\left(g^{-1} h\right)=0$, because $g^{-1} h \in Z$. Since the action respects the grading, each homogeneous degree $d$ part $I(Z)_{d}$ is a $\mathrm{GL}_{m}$-representation. The coordinate ring $\mathbb{C}[Z]$ is defined as the quotient algebra $\mathbb{C}\left[\mathbb{A}_{m}^{n}\right] / I(Z)$ and each homogeneous part $\mathbb{C}[Z]_{d}=\mathbb{C}\left[\mathbb{A}_{m}^{n}\right]_{d} / I(Z)_{d}$ is a $\mathrm{GL}_{m}$-representation. Equivalently, we can define $\mathbb{C}[Z]$ as the set of restrictions of functions in $\mathbb{C}\left[\mathbb{A}_{m}^{n}\right]$ to $Z$.

For most sets of parameters we have $\operatorname{Pow}_{m, k}^{n} \nsubseteq \mathbb{\mathrm { Ch } _ { m } ^ { n }}$, but there are some exceptions. Clearly Pow ${ }_{m, 1}^{n} \subseteq \mathrm{Ch}_{m}^{n}$. Moreover, $\operatorname{Pow}_{1, k}^{n}=\mathrm{Ch}_{1}^{n}$ for all $n \geq 1, k \geq 1$; and $\operatorname{Pow}_{m, k}^{1}=\mathrm{Ch}_{m}^{1}$ for all $m \geq 1, k \geq 1$. It is also easy to see that $\operatorname{Pow}_{2,2}^{2} \subseteq \mathrm{Ch}_{2}^{2}$, because $\ell_{1}^{2}+\ell_{2}^{2}=\left(\ell_{1}+i \ell_{2}\right)\left(\ell_{1}-i \ell_{2}\right)$, where $i^{2}=-1$. More generally, $\left(\ell_{1}+\zeta \ell_{2}\right)\left(\ell_{1}+\zeta^{2} \ell_{2}\right) \cdots\left(\ell_{1}+\zeta^{n} \ell_{2}\right)=\ell_{1}^{n}+\zeta^{\frac{n(n+1)}{2}} \ell_{2}^{n}$ for $\zeta^{n}=1$, which implies $\operatorname{Pow}_{m, 2}^{n} \subseteq \mathrm{Ch}_{m}^{n}$. For $m=2, k \geq 1, n \geq 1$, we have $\operatorname{Pow}_{m, k}^{n} \subseteq \mathrm{Ch}_{m}^{n}$ by the fundamental theorem of algebra. These are the only exceptions, as for $n \geq 2, m \geq 3$, $k \geq 3$ we have $\operatorname{Pow}_{m, k}^{n} \not \subset \mathrm{Ch}_{m}^{n}$ : the polynomial $x^{n}+y^{n}+z^{n}$ of the Fermat curve is in $\operatorname{Pow}_{m, k}^{n}$ and its irreducibility implies (since $n \geq 2$ ) that $x^{n}+y^{n}+z^{n} \notin \mathrm{Ch}_{m}^{n}$.

We will see that for specific settings of parameters there exist multiplicity obstructions that prove $\mathrm{Pow}_{m, k}^{n} \nsubseteq \mathrm{Ch}_{m}^{n}$, but there do not exist occurrence obstructions that prove this fact (see the definitions below). Our approach works as follows and is in complete analogy to the approach proposed in $[22,23]$ to separate group varieties arising from algebraic complexity theory. If $\mathrm{Pow}_{m, k}^{n} \subseteq \mathrm{Ch}_{m}^{n}$, then the restriction of functions gives a canonical $\mathrm{GL}_{m}$-equivariant surjection $\mathbb{C}\left[\mathrm{Ch}_{m}^{n}\right]_{d} \rightarrow \mathbb{C}\left[\mathrm{Pow}_{m, k}^{n}\right]_{d}$. In this case, Schur's lemma (e.g. [11, Lemma 4.1.4]) implies that

$$
\operatorname{mult}_{\lambda}\left(\mathbb{C}\left[\mathrm{Ch}_{m}^{n}\right]_{d}\right) \geq \operatorname{mult}_{\lambda}\left(\mathbb{C}\left[\mathrm{Pow}_{m, k}^{n}\right]_{d}\right) .
$$

for all $m$-partitions $\lambda$. Therefore, a partition $\lambda$ that violates (2) proves that $\operatorname{Pow}_{m, k}^{n} \not \subset \mathrm{Ch}_{m}^{n}$. Such a $\lambda$ is called a multiplicity obstruction. If additionally mult $\lambda\left(\mathbb{C}\left[\mathrm{Ch}_{m}^{n}\right]_{d}\right)=0$, then $\lambda$ is called an occurrence obstruction.

Since $\mathrm{Ch}_{m}^{n}$ and $\mathrm{Pow}_{m, k}^{n}$ are subvarieties of $\mathbb{A}_{m}^{n}$ and since all $\lambda$ for which $\operatorname{mult}_{\lambda}\left(\mathbb{C}\left[\mathbb{A}_{m}^{n}\right]_{d}\right)>0$ are $m$-partitions of $d n$, it follows that if $\operatorname{mult}_{\lambda}\left(\mathbb{C}\left[\mathrm{Ch}_{m}^{n}\right]_{d}\right)>0$ or $\operatorname{mult}_{\lambda}\left(\mathbb{C}\left[\mathrm{Pow}_{m, k}^{n}\right]_{d}\right)>0$, then $\lambda$ is an $m$-partition of $d n$.

- Theorem 2.1 (Main Theorem).

(1) Asymptotic result: Let $m \geq 3, n \geq 2, k=d=n+1, \lambda=\left(n^{2}-2, n, 2\right)$. We have mult $_{\lambda}\left(\mathbb{C}\left[\mathrm{Ch}_{m}^{n}\right]_{d}\right)<\operatorname{mult}_{\lambda}\left(\mathbb{C}\left[\mathrm{Pow}_{m, k}^{n}\right]_{d}\right)$, i.e., $\lambda$ is a multiplicity obstruction that shows Pow $_{m, k}^{n} \nsubseteq \mathrm{Ch}_{m}^{n}$.

(2) Finite result: In two finite settings we can show a slightly stronger separation:

(a) Let $k=4, n=6, m=3, d=7, \lambda=\left(n^{2}-2, n, 2\right)=(34,6,2)$. Then $\operatorname{mult}_{\lambda}\left(\mathbb{C}\left[\mathrm{Ch}_{m}^{n}\right]_{d}\right)=7<8=\operatorname{mult}_{\lambda}\left(\mathbb{C}\left[\mathrm{Pow}_{m, k}^{n}\right]_{d}\right)$, i.e., $\lambda$ is a multiplicity obstruction that shows Pow $_{m, k}^{n} \not \subset \mathrm{Ch}_{m}^{n}$.

(b) Similarly, for $k=4, n=7, m=4, d=8, \lambda=\left(n^{2}-2, n, 2\right)=(47,7,2)$ we have mult $_{\lambda}\left(\mathbb{C}\left[\mathrm{Ch}_{m}^{n}\right]_{d}\right)<11=\operatorname{mult}_{\lambda}\left(\mathbb{C}\left[\mathrm{Pow}_{m, k}^{n}\right]_{d}\right)$, i.e., $\lambda$ is a multiplicity obstruction that shows $\operatorname{Pow}_{m, k}^{n} \not \subset \mathrm{Ch}_{m}^{n}$.

Both separations (a) and (b) cannot be achieved using occurrence obstructions, even for arbitrary $k$ : for all m-partitions $\mu$ that satisfy $a_{\mu}(d[n])>0$ we have mult $_{\lambda}\left(\mathbb{C}\left[\mathrm{Ch}_{m}^{n}\right]_{d^{\prime}}\right)>0$ in these settings. 
One would like to show that there are no occurrence obstructions in all cases (1), but this is wrong if $n$ is not large enough with respect to $m$, see Prop. 3.11. Even for $m=3$ or $m=4$ ruling out occurrence obstructions as in (2) is done by a large-scale computer calculation which is only suitable for a finite case, but not for sequences as in (1). The papers $[16,8]$ rule out occurrence obstructions for families, but only in ranges where they would give very strong new algebraic circuit lower bounds, so that we expect it to be difficult to find multiplicity obstructions in those cases. Note also that $[16,8]$ are only dealing with padded polynomials, for which [17] guarantees $\lambda$ to have a very restricted shape.

We expect multiplicity obstructions to be more powerful than occurrence obstructions in most cases relevant for geometric complexity theory, and Theorem 2.1 resolves the challenge of finding a setting in which the corresponding multiplicities and occurrences could actually be computed in a reasonable amount of time, while the setting is also involved enough so that a difference between occurrence obstructions and multiplicity obstructions could be witnessed.

Remark 2.2. The partition $\left(n^{2}-2, n, 2\right)$ is known to be the type of one of Brill's classical set-theoretic equations for $\mathrm{Ch}_{m}^{n}$, see [12].

\section{Proof of the main theorem}

The main theorem (Theorem 2.1) makes a statement about the finite situations $k=4, n=6$, $m=3, d=7$ and $k=4, n=7, m=4, d=8$, as well as the general situation $m \geq 3, n \geq 2$, $k=d=n+1$. As a first step, in all these cases we show that

$$
\operatorname{mult}_{\lambda}\left(\mathbb{C}\left[\operatorname{Pow}_{m, k}^{n}\right]_{d}\right)=a_{\lambda}(d[n]) .
$$

In the finite cases the following computer calculation suffices to prove (3).

- Proposition 3.1. $\operatorname{mult}_{(34,6,2)}\left(\mathbb{C}\left[\mathrm{Pow}_{3,4}^{6}\right]_{7}\right)=8=a_{(34,6,2)}(7[6])$ and $\operatorname{mult}_{(47,7,2)}\left(\mathbb{C}\left[\operatorname{Pow}_{3,4}^{7}\right]_{8}\right)=$ $11=a_{(47,7,2)}(8[7])$.

Proof. The plethysm coefficient computations were performed with the LIE software. The rest is a small computer calculation completely analogous to the ones in [8, Sec. 6]. The details can be found in the full version of this paper.

For the general situation the equality (3) is a consequence of the following result on power sums proved in [8, Prop. 3.2]:

- Proposition 3.2. If $\lambda$ is an m-partition of $d n$ and $k \geq d$, then $\operatorname{mult}_{\lambda}\left(\mathbb{C}\left[\operatorname{Pow}_{m, k}^{n}\right]_{d}\right)=$ $a_{\lambda}(d[n])$.

As a second step we will use the following lemma for $\lambda=\left(n^{2}-2, n, 2\right)$.

Lemma 3.3 (see also [19, Sec. 9.2.3]). Let $\lambda$ be an m-partition and $n \geq m$. Then $\operatorname{mult}_{\lambda}\left(\mathbb{C}\left[\mathrm{Ch}_{m}^{n}\right]_{d}\right) \leq a_{\lambda}(n[d])$.

Proof. Let $\mathrm{GL}_{n}\left(x_{1} \cdots x_{n}\right):=\left\{g\left(x_{1} \cdots x_{n}\right) \mid g \in \mathrm{GL}_{n}\right\} \subseteq \mathbb{A}_{n}^{n}$ denote the $\mathrm{GL}_{n}$-orbit of $x_{1} \cdots x_{n}$. We denote by $\overline{\mathrm{GL}_{n}\left(x_{1} \cdots x_{n}\right)}$ the Zariski closure of this orbit, which equals its Euclidean closure by the same principles as in Section 2. Choose bases and embed $\mathbb{A}_{m}^{n} \subseteq \mathbb{A}_{n}^{n}$, so that $\mathrm{Ch}_{m}^{n}$ is the intersection of $\mathbb{A}_{m}^{n}$ and $\overline{\mathrm{GL}_{n}\left(x_{1} \cdots x_{n}\right)}$. This implies (via argument analogous to that for the plethysm coefficient $\left(\left[14\right.\right.$, Lem. 4.3.2])) that the multiplicity of the irreducible $\mathrm{GL}_{m^{-}}$ representation $\{\lambda\}_{m}$ in $\mathbb{C}\left[\mathrm{Ch}_{m}^{n}\right]_{d}$ equals the multiplicity of the irreducible $\mathrm{GL}_{n}$-representation $\{\lambda\}_{n}$ in $\mathbb{C}\left[\overline{\mathrm{GL}_{n}\left(x_{1} \cdots x_{n}\right)}\right]$. In other words mult ${ }_{\lambda}\left(\mathbb{C}\left[\mathrm{Ch}_{m}^{n}\right]_{d}\right)=\operatorname{mult}_{\lambda}\left(\mathbb{C}\left[\overline{\mathrm{GL}_{n}\left(x_{1} \cdots x_{n}\right)}\right]_{d}\right)$. The vector space $\mathbb{C}\left[\overline{\mathrm{GL}_{n}\left(x_{1} \cdots x_{n}\right)}\right]_{d}$ consists of exactly the restrictions of polynomials in $\mathbb{C}\left[\mathbb{A}_{n}^{n}\right]_{d}$ to 
the orbit $\mathrm{GL}_{n}\left(x_{1} \cdots x_{n}\right)$. The coordinate ring $\mathbb{C}\left[\mathrm{GL}_{n}\left(x_{1} \cdots x_{n}\right)\right]$ is also graded and its homogeneous degree $d$ part $\mathbb{C}\left[\mathrm{GL}_{n}\left(x_{1} \cdots x_{n}\right)\right]_{d}$ consists of all homogeneous degree $d$ regular functions on $\mathrm{GL}_{n}\left(x_{1} \cdots x_{n}\right)$, in particular mult ${ }_{\lambda}\left(\mathbb{C}\left[\overline{\mathrm{GL}_{n}\left(x_{1} \cdots x_{n}\right)}\right]_{d}\right) \leq \operatorname{mult}_{\lambda}\left(\mathbb{C}\left[\mathrm{GL}_{n}\left(x_{1} \cdots x_{n}\right)\right]_{d}\right)$. The right-hand side can be understood via geometric invariant theory as follows (see [14, Sec. 3.4(A)]): $\operatorname{mult}_{\lambda}\left(\mathbb{C}\left[\mathrm{GL}_{n}\left(x_{1} \cdots x_{n}\right)\right]_{d}\right)=\operatorname{mult}_{\lambda^{*}}\left(\mathbb{C}\left[\mathrm{GL}_{n}\right]_{d}^{H}\right)$, where $H=\left\{\operatorname{diag}\left(\alpha_{1}, \ldots, \alpha_{n}\right) \mid\right.$ $\left.\prod_{i=1}^{n} \alpha_{1}=1\right\} \rtimes \mathfrak{S}_{n} \subseteq \mathrm{GL}_{n}$ is the stabilizer of $x_{1} \cdots x_{n}$. The algebraic Peter-Weyl theorem (see e.g. [18, II.3.1 Satz 3], [11, Thm. 4.2.7], or [27, Ch. 7, 3.1 Thm.]) states that $\mathbb{C}\left[\mathrm{GL}_{n}\right]=\bigoplus_{\lambda}\{\lambda\} \otimes\left\{\lambda^{*}\right\}$ and we conclude $\operatorname{mult}_{\lambda}\left(\mathbb{C}\left[\mathrm{GL}_{n}\right]_{d}^{H}\right)=\operatorname{dim}\{\lambda\}^{H}$. There are several ways of seeing that $\operatorname{dim}\{\lambda\}^{H}=a_{\lambda}(n[d])$, see e.g. [19, Sec. 9.2.3] or [15, Prop. 3.3]. This proves the lemma.

Now an argument using symmetric functions is used to prove the following theorem.

- Theorem 3.4. $a_{\left(n^{2}-2, n, 2\right)}(n+1[n])=1+a_{\left(n^{2}-2, n, 2\right)}(n[n+1])$.

Theorem 3.4 is a corollary of more general results, see Corollary 4.4 in the appendix.

This finishes the proof that $\left(n^{2}-2, n, 2\right)$ is a multiplicity obstruction in all cases of Theorem 2.1.

\section{No occurrence obstructions}

To finish the proof of Theorem 2.1(2), it remains to show that there are no occurrence obstructions in the finite situation $n=6, m=3$ and $n=7, m=4$. We will primarily go into more detail for the first case and the second one will be proven similarly. We will do this by showing that

$$
a_{\mu}(d[n])>0 \text { implies } \operatorname{mult}_{\mu}\left(\mathbb{C}\left[\mathrm{Ch}_{m}^{n}\right]_{d}\right)>0 \text { for } n=6, m=3 .
$$

Note that this claim is independent of $k$. We start proving (4) by giving a complete classification of when $a_{\mu}(d[n])>0$ for the case $n=6, m=3$.

First, the following lemma states that for a few special $\mu$ the plethysm coefficient always vanishes.

- Lemma 3.5. Let $\bar{\lambda}:=\left(\lambda_{2}, \lambda_{3}, \ldots\right)$ denote $\lambda$ without its first row. If $\lambda$ is an m-partition of $d n$ and $\bar{\lambda} \in\{(3,3),(3,1),(2,1),(1,1),(1)\}$, then $a_{\lambda}(d[n])=0$.

Proof. This is proved by a finite calculation for all cases but $(3,3)$ as Thm 1.10 (a) in [16]. Exactly the same calculation can be used to also prove the result for the additional partition $(3,3)$.

For characterizing the set of all $\mu$ for which $a_{\mu}(d[n])$ is positive, we observe that they form a finitely generated semigroup and hence we only need to find the semigroup's generators:

$$
\text { If } a_{\mu}(d[n])>0 \text { and } a_{\nu}\left(d^{\prime}[n]\right)>0 \text {, then } a_{\mu+\nu}\left(d+d^{\prime}[n]\right)>0 \text {. }
$$

A detailed proof of (5) can be found for example in [1, Prop. 21.2.6].

- Proposition 3.6. Define the set

$X:=\{(6),(6,6),(8,4),(10,2),(6,6,6),(8,6,4),(10,4,4),(9,6,3),(8,8,2),(10,6,2),(11,5,2)$, $(10,7,1),(12,4,2),(11,6,1),(10,8),(14,2,2),(13,4,1),(13,5),(15,3),(8,8,8),(10,8,6),(11,7,6)$, $(10,9,5),(11,8,5),(10,10,4),(12,7,5),(11,9,4),(13,6,5),(12,8,4),(11,10,3),(13,7,4),(12,9,3)$, $(13,8,3),(12,10,2),(15,5,4),(14,7,3),(13,9,2),(13,10,1),(16,5,3),(15,7,2),(14,9,1),(17,4,3)$, $(15,8,1),(15,9),(19,3,2),(18,5,1),(17,7),(10,10,10),(11,10,9),(12,10,8),(13,9,8),(12,11,7)$, $(13,10,7),(14,9,7),(13,11,6),(15,8,7),(13,12,5),(16,7,7),(15,9,6),(14,11,5),(13,13,4),(15,10,5)$, $(15,11,4),(14,13,3),(16,11,3),(15,13,2),(15,14,1),(17,13),(13,12,11),(14,11,11),(13,13,10)$, $(15,11,10),(14,13,9),(16,11,9),(15,13,8),(15,14,7),(18,9,9),(15,15,6),(17,17,2),(18,17,1)$, $(26,5,5),(15,14,13),(16,13,13),(15,15,12),(17,17,8),(18,15,15),(17,17,14),(25,23),(45,45)\}$.

Here we truncated trailing zeros from the 3-partitions. The set $X$ is the set of generators of the semigroup of 3-partitions $\mu$ that have $a_{\mu}(d[6])>0$. 
The proof of Proposition 3.6 proceeds in several steps.

A direct computation with the LIE software verifies $a_{\mu}(d[6])>0$ for all $\mu \in X \backslash\{(45,45)\}$. The case $d=15$ runs into memory problems when using LIE. Other software such as ScHUR stops working when $d=8$. We used the formula [32, Cor. 4.2.8] to verify $a_{(45,45)}(15[6])>0$.

We call the number of nonzero parts the length of a partition. We use a brute-force computer verification and a direct computation with LIE to show that for $d \leq 26$ every partition $\mu$ of length $\leq 2$ with $a_{\mu}(d[6])>0$ is a sum of partitions from the set $X$. The same computation is done for all 3 -partitions, but only up to $d \leq 14$. The following proposition states that these finite computations completely describe all cases.

Proposition 3.7. If $\lambda$ is a 3-partition of $6 d, d \geq 15$, and $\bar{\lambda} \notin\{(3,3),(3,1),(2,1),(1,1),(1)\}$, then $\lambda$ is a sum of partitions from $X$.

Proof. For $15 \leq d \leq 17$ we use a computer calculation to show that we can write every such partition $\lambda$ as a sum of partitions from $X$. For $d>17$ we prove this inductively by showing that we can write every 3 -partition $\lambda$ of $6 d$ with $\bar{\lambda} \notin\{(3,3),(3,1),(2,1),(1,1),(1)\}$ as a sum of one of the partitions $(6),(6,6)$ or $(6,6,6)$ and a smaller $\lambda^{\prime}$ with again $\bar{\lambda}^{\prime} \notin$ $\{(3,3),(3,1),(2,1),(1,1),(1)\}$.

Let $c_{i}$ denote the number of columns in $\lambda$ with exactly $i$ boxes for $i \in\{1,2,3\}$. Since we have at least 108 boxes in $\lambda$, the pigeonhole principle implies that at least one must be true: $c_{1} \geq 6, c_{2} \geq 10$ or $c_{3} \geq 10$.

In the case $c_{1} \geq 6$ we have $\lambda=\lambda^{\prime}+(6)$ with $\lambda^{\prime}$ being a sum of elements from $X$ since $\bar{\lambda}^{\prime}=\bar{\lambda}$. In the case $c_{2} \geq 10$ we have $\lambda=\lambda^{\prime}+(6,6)$ with $\lambda^{\prime}$ being a sum of elements from $X$ as $\lambda_{2}^{\prime} \geq 4$. In the case $c_{3} \geq 10$ we have $\lambda=\lambda^{\prime}+(6,6,6)$ with $\lambda^{\prime}$ being a sum of elements from $X$ as $\lambda_{3}^{\prime} \geq 4$.

This finishes the proof of Proposition 3.6.

To prove (4) it is sufficient (and necessary) to show that mult $\mu\left(\mathbb{C}\left[\mathrm{Ch}_{m}^{n}\right]_{d}\right)>0$ for all $\mu \in X$, because a semigroup property analogous to (5) holds (the same proof applies, e.g. [1, Prop. 21.2.6]):

$$
\text { If } \operatorname{mult}_{\mu}\left(\mathbb{C}\left[\mathrm{Ch}_{m}^{n}\right]_{d}\right)>0 \text { and mult }{ }_{\nu}\left(\mathbb{C}\left[\mathrm{Ch}_{m}^{n}\right]_{d^{\prime}}\right)>0 \text {, then mult }{ }_{\mu+\nu}\left(\mathbb{C}\left[\mathrm{Ch}_{m}^{n}\right]_{d+d^{\prime}}\right)>0 \text {. }
$$

If the length of $\mu$ is at most 2 , we use the following general result.

- Proposition 3.8. Let $\mu$ be a 3-partition of length at most 2. If $a_{\mu}(d[n])>0$, then $\operatorname{mult}_{\mu}\left(\mathbb{C}\left[\mathrm{Ch}_{m}^{n}\right]_{d}\right)>0$.

Proof. We use an inheritance result: If for a 2-partition $\mu$ we have mult $\mu\left(\mathbb{C}\left[\mathrm{Ch}_{2}^{n}\right]_{d}\right)>0$, and $\nu$ is the 3 -partition that arises from $\mu$ by adding a single 0 , then $\operatorname{mult}_{\nu}\left(\mathbb{C}\left[\mathrm{Ch}_{3}^{n}\right]_{d}\right)>0$. The proof is completely analogous to other inheritance results, see e.g. [14, Lemma 4.3.2 or Sec. 5.3]. Now for 2-partitions $\mu$ we have $a_{\mu}(d[n])=\operatorname{mult}_{\mu}\left(\mathbb{C}\left[\mathrm{Ch}_{2}^{n}\right]_{d}\right)$, because every homogeneous polynomial in 2 variables decomposes as a product of homogeneous linear polynomials by the fundamental theorem of algebra, see also e.g. [19, Exa. 9.1.1.8]. This is how the Hermite reciprocity can be proved. An even simpler argument works if $\mu$ has length 1 .

We finish the proof of (4) by using a computer calculation to verify that for all 3-partitions $\mu \in X$ of length 3 we have $\operatorname{mult}_{\mu}\left(\mathbb{C}\left[\mathrm{Ch}_{3}^{6}\right]\right)>0$, see Proposition 5.1.

This finishes the proof of Theorem $2.1(2 \mathrm{a})$. The proof of Theorem $2.1(2 \mathrm{~b})$ is completely analogous as follows. Let $m=4, n=7$. 
- Lemma 3.9. Let $\bar{\lambda}:=\left(\lambda_{2}, \lambda_{3}, \ldots\right)$ denote $\lambda$ without its first row. If $\lambda$ is an m-partition of $d n$ and $\bar{\lambda} \in Y$ for $Y:=\{(1),(1,1),(1,1,1),(2,1),(2,1,1),(2,2,1),(3,1),(3,1,1)$, $(3,2,1),(3,3),(3,3,1),(3,3,2),(3,3,3),(4,1,1),(4,3,3),(5,1,1),(5,5,5),(6,1,1)\}$, then $a_{\lambda}(d[n])=0$.

Proof. This is proven exactly like Lemma 3.5.

The semigroup of 4-partitions $\lambda$ that have $a_{\lambda}(d[7])>0$ has 948 generators, listed in the full version of this paper. They form a set that we call $X$.

We again use a direct computation with the LIE software to verify $a_{\mu}(d[7])>0$ for all $\mu \in X \backslash\{(49,49),(24,24,23,23)\}$. For both the remaining partitions $\mu \in\{(49,49),(24,24,23$, $23)\}$ we prove mult ${ }_{\mu}\left(\mathbb{C}\left[\mathrm{Ch}_{4}^{7}\right]_{d}\right)>0$ using our computer calculations which also implies $a_{\mu}(d[7])>0$.

To prove those are all the generators we use the following proposition which is proved completely analogously to Proposition 3.7.

- Proposition 3.10. If $\lambda$ is a 4-partition of $7 d, d \geq 14$, and $\bar{\lambda} \notin Y$, then $\lambda$ is a sum of partitions from $X$.

For the next finite case $(n=7, k=d=8, m=5)$ we reached the computational limit of our implementation. Here we were able to find 5016 generating partitions of the semigroup of 4-partitions $\mu$ that have $a_{\mu}(d[7])>0$. Unfortunately these do not generate everything excluding the exceptions yet. We were able to verify for 5000 generating partitions $\mu$ that $\operatorname{mult}_{\mu}\left(\mathbb{C}\left[\mathrm{Ch}_{m}^{n}\right]_{d}\right)>0$. For the remaining ones, we used up to $200 \mathrm{~GB}$ of RAM, but this was not sufficient.

\section{Some occurrence obstructions}

As we degenerate the parameter settings and let $n$ get closer to $m$, multiplicity obstructions tend to become occurrence obstructions. More precisely, for $m=3$ and values of $n<6$, and for $(m, n)=(4,6)$, some multiplicity obstructions are actually also occurrence obstructions, as the following proposition shows.

Proposition 3.11. The following partitions give occurrence obstructions that show Pow $_{m, d}^{n} \nsubseteq \mathrm{Ch}_{m}^{n}$.

\begin{tabular}{c|c|c|c|c|c}
$m$ & $n$ & $\lambda$ & $d$ & $a_{\lambda}(d[n])$ & $a_{\lambda}(n[d])$ \\
\hline 3 & 2 & $(2,2,2)$ & 3 & 1 & 0 \\
3 & 3 & $(7,3,2)$ & 4 & 1 & 0 \\
3 & 4 & $(11,9,8)$ & 7 & 1 & 0 \\
3 & 5 & $(12,9,9)$ & 6 & 1 & 0 \\
4 & 6 & $(14,14,13,13)$ & 9 & 11 & 0
\end{tabular}

Proof. The plethysm coefficient computations were performed with the LIE software. Lemma 3.3 implies that $\operatorname{mult}_{\lambda}\left(\mathbb{C}\left[\mathrm{Ch}_{m}^{n}\right]_{d}\right) \leq a_{\lambda}(n[d])=0$. Proposition 3.2 implies $\operatorname{mult}_{\lambda}\left(\mathbb{C}\left[\operatorname{Pow}_{m, d}^{n}\right]_{d}\right)>0$.

See [4, Prop. 4] for additional occurrence obstructions in the case $n=3$. 


\section{$4 \quad$ Plethysm inequalities}

We are interested in the plethysm coefficients $a_{\lambda}(d[m])$ for certain values of $\lambda$ and $d, m$. Here we compute such values for infinite families of parameters and in particular, prove Theorem 3.4.

We will work over the ring of symmetric functions $\Lambda$, defined as the ring of formal power series (in finitely or infinitely many variables) which are invariant under any transposition of the variables. For the definitions and main identities see e.g. [29]. Plethysms of symmetric functions are described also there in Appendix 2 of Chapter 7, here we review the necessary definitions.

The characters of the irreducible $G L_{r}$-module $W_{\lambda}$ are the Schur functions $s_{\lambda}\left(x_{1}, \ldots, x_{r}\right)$, where $x_{1}, \ldots, x_{r}$ correspond to the eigenvalues of the conjugacy class representative from $G L_{r}$. Their combinatorial interpretation is as the generating function over all semi-standard Young tableaux with entries $1, \ldots, r$, but we will use certain determinantal formulas as described below. The complete homogeneous symmetric functions $h_{\ell}$ are defined as $s_{(\ell)}$ and are the characters of the $\operatorname{Sym}^{\ell}$ module. The $\operatorname{Sym}^{d}\left(\operatorname{Sym}^{n}\left(\mathbb{C}^{r}\right)\right)$ module is obtained as the composition of the two representations. The image in $\operatorname{Sym}^{n}\left(\mathbb{C}^{r}\right)$ of a diagonal matrix from $G L_{r}$ with entries (i.e. eigenvalues) $x_{1}, \ldots, x_{r}$ on the diagonal has eigenvalues all the $N:=\left(\begin{array}{c}n+r-1 \\ r-1\end{array}\right)$ degree $n$ monomials in $x_{1}, \ldots, x_{r}$. Hence, the character of the representation $\operatorname{Sym}^{d}\left(\operatorname{Sym}^{n}\left(\mathbb{C}^{r}\right)\right)$ of $G L_{N}$ can be obtained by evaluating the character $h_{d}$ of Sym ${ }^{d}$ at the monomials, i.e. the eigenvalues above. This gives us the definition of the symmetric function plethysm $h_{d}\left[h_{n}\left(x_{1}, \ldots, x_{r}\right)\right]$, that is, the evaluation of $h_{d}$ on the variables consisting of all degree $n$ monomials, i.e. $h_{d}\left[h_{n}\left(x_{1}, \ldots, x_{r}\right)\right]:=h_{d}\left(x_{1}^{n}, x_{1}^{n-1} x_{2}, x_{1}^{n-1} x_{3}, \ldots, x_{1}^{\alpha_{1}} \cdots x_{r}^{\alpha_{r}}, \ldots\right)$, where $\alpha=\left(\alpha_{1}, \ldots, \alpha_{r}\right)$ runs over all compositions of $n$.

In general, knowing the character of a representation contains all the information to obtain the multiplicities of the irreducible decomposition via the inner product of characters. As the Schur functions $s_{\lambda}$ are the irreducible characters for $G L_{r}$, the inner product is equivalent to an inner product in the ring $\Lambda$, where $\left\{s_{\lambda}\right\}_{\lambda}$ is an orthonormal basis. In other words, the multiplicity of the Weyl module of weight $\lambda$ is given by the multiplicity of the Schur function $s_{\lambda}$ in the expansion of $h_{d}\left[h_{m}\right]$. We will now compute this via the inner product in the ring $\Lambda$ of symmetric functions, using some basic properties of this ring as found in [29] and [21].

We have that $a_{\lambda}(d[n])$ is the multiplicity of $\{\lambda\}$ in $\mathrm{Sym}^{d} \mathrm{Sym}^{n}$, translated into characters this is also the coefficient at $s_{\lambda}$ of the expansion of $h_{d}\left[h_{n}\right]$ in Schur function. By their orthonormalitiy, this is the same as

$$
a_{\lambda}(d[n])=\left\langle s_{\lambda}, h_{d}\left[h_{n}\right]\right\rangle
$$

We now invoke various symmetric function identities in order to compute the above inner product. The Schur functions $s_{\lambda}$ can be expressed via the Jacobi-Trudi formula (see again [29, Ch. 7]) as a signed sums of homogeneous symmetric functions, namely

$$
s_{\lambda}=\operatorname{det}\left[h_{\lambda_{i}-i+j}\right]_{i, j=1}^{\ell(\lambda)},
$$

the inner product (7) can then be computed via a signed sum of inner products of the form $\left\langle h_{\mu}, h_{d}\left[h_{n}\right]\right\rangle$. We remark that the orthogonal dual basis for the complete homogeneous symmetric functions is the monomial symmetric functions, i.e. $\left\langle h_{\mu}, m_{\nu}\right\rangle=\delta_{\mu, \nu}$, so we need to express $h_{d}\left[h_{n}\right]$ in terms of the monomial symmetric functions, defined by

$$
m_{\nu}\left(x_{1}, \ldots, x_{r}\right):=\sum_{\sigma \in S_{r}(\nu)} x_{1}^{\nu_{\sigma(1)}} x_{2}^{\nu_{\sigma(2)}} \cdots x_{r}^{\nu_{\sigma(r)}},
$$


where the sum ranges over all distinct permutations of $\left(\nu_{1}, \nu_{2}, \ldots, \nu_{r}\right)$ and $\nu$ is completed with 0 s to the length $r$. Since he monomial symmetric functions form a basis for $\Lambda$, we can expand any symmetric function in it uniquely. Let $h_{d}\left[h_{m}\right]=\sum_{\nu} c_{\nu} m_{\nu}$, for some constants $c_{\nu}$ (i.e. the coefficients in this expansion). Since each $m_{\nu}$ has a unique leading monomial (in the lexicographic order) $x_{1}^{\nu_{1}} x_{2}^{\nu_{2}} \cdots$, finding $c_{\nu}$ is equivalent to extracting the coefficient at the single monomial $x_{1}^{\nu_{1}} \cdots$ from the monomial expansion of the corresponding symmetric function as a polynomial, i.e. $c_{\nu}=\left(x_{1}^{\nu_{1}} x_{2}^{\nu_{2}} \ldots\right) @ h_{d}\left[h_{n}\left(x_{1}, x_{2}, \ldots\right)\right]$, where to avoid confusion with the plethysm notation we denote by $(X) @ f$ the coefficient of the monomial $X$ in the monomial expansion of the polynomial $f$.

Let $\nu$ be a partition of length $\ell$. By the above remarks we need to consider only the truncated expansion $h_{d}\left[h_{n}\left(x_{1}, \ldots, x_{\ell}\right)\right]$ as only the monomials in $x_{1}, \ldots, x_{\ell}$ will be relevant.

We have the following formula for the $h$ 's, see e.g. [29]:

$$
h_{N}\left(x_{1}, \ldots, x_{r}\right)=\sum_{(b): b_{1}+b_{2}+\cdots=N} x_{1}^{b_{1}} x_{2}^{b_{2}} \cdots,
$$

where $(b)=\left(b_{1}, b_{2}, \ldots, b_{r}\right)$ runs over all (weak) compositions of $N$. Hence, assuming some total ordering for compositions $\alpha^{i}$ of $n$, we have

$$
h_{d}\left[h_{n}\left(x_{1}, \ldots, x_{r}\right)\right]=h_{d}\left[\ldots, x^{\alpha^{i}}, \ldots\right]=\sum_{(b):|b|=d} x^{\sum_{i} b_{i} \alpha^{i}} .
$$

Thus for the coefficients $c_{\nu}$ we have:

$$
c_{\nu}(d, n):=\left(x^{\nu}\right) @ h_{d}\left[h_{n}\right]=\left\langle h_{\nu}, h_{d}\left[h_{n}\right]\right\rangle=\#\left\{(b):|b|=d, \sum_{i} b_{i} \alpha^{i}=\nu\right\}
$$

By the Jacobi-Trudi identity (8) this gives a formula for computing the plethysm coeff. as

$$
a_{\lambda}(d[n])=\left\langle\operatorname{det}\left[h_{\lambda_{i}-i+j}\right]_{i, j=1}^{\ell(\lambda)}, h_{d}\left[h_{n}\right]\right\rangle=\sum_{\pi \in S_{\ell(\lambda)}} \operatorname{sgn}(\pi) c_{\lambda+\pi-(1,2, \ldots)}(d, n),
$$

where the permutations $\pi$ are viewed as vectors with entries $1,2, \ldots, \ell(\lambda)$

We now turn towards the proof of Theorem 3.4 and consider $s_{\lambda}$ for $\lambda=\left(\lambda_{1}, \lambda_{2}, 2\right)$ for some $k \geq 2$. By the Jacobi-Trudi identity (10) we need to compute only $c_{\nu}$ for $\nu$ having at most 3 parts, with $\nu_{3}=0,1,2$. Let $p_{r}(a, b)$ denote the number of partitions of $r$ which fit inside an $a \times b$ rectangle, it's generating function is the $q$-binomial coefficient (see [31]): $\left(\begin{array}{c}a+b \\ a\end{array}\right)_{q}=\frac{(1-q) \cdots\left(1-q^{a+b}\right)}{(1-q) \cdots\left(1-q^{a}\right)(1-q) \cdots\left(1-q^{b}\right)}=\sum_{r=0}^{a b} p_{r}(a, b) q^{r}$

- Proposition 4.1. We have the following generating function identities for $c_{\nu}(d, n)$, where $\ell(\nu) \leq 3$ and $\nu_{3} \leq 2$ :

$$
\begin{aligned}
& \left.c_{(L, k, 2)}=\left(q^{k}\right) @\left(\begin{array}{c}
n \\
1
\end{array}\right)_{q}\left(\begin{array}{c}
n+d-2 \\
n
\end{array}\right)_{q}+\left(\begin{array}{c}
n-1 \\
1
\end{array}\right)_{q}\left(\begin{array}{c}
n+d-1 \\
n
\end{array}\right)_{q}+q\left(\begin{array}{c}
n \\
2
\end{array}\right)_{q}\left(\begin{array}{c}
n+d-2 \\
n
\end{array}\right)_{q}\right) \\
& c_{(L, k, 1)}=\left(q^{k}\right) @\left(\begin{array}{c}
n \\
1
\end{array}\right)_{q}\left(\begin{array}{c}
n+d-1 \\
n
\end{array}\right)_{q} \\
& c_{(L, k, 0)}=\left(q^{k}\right) @\left(\begin{array}{c}
n+d \\
n
\end{array}\right)_{q}=p_{k}(n, d)
\end{aligned}
$$

Proof. By formula (9), we have $c_{(L, k, 0)}=\#\left\{(b):|b|=d, \sum b_{i} \alpha^{i}=(L, k)\right\}$.

Hence, the only $\alpha^{i}$ involved are of the form $\alpha^{i}=\left(n-a_{i}, a_{i}\right)$, and after renumerating, we can assume $a_{i}=i$. So we are counting compositions $b$ of $d$, s.t. $\sum_{i} b_{i} i=k$ for $i=0 \ldots n$. This is exactly the same as specifying an integer partition $\gamma$ of $k$ by the number of its parts, i.e. $\gamma=\left(0^{b_{0}}, 1^{b_{1}}, \ldots, n^{b_{n}}\right)$, such that $b_{0}+\cdots+b_{n}=d$. These restrictions are equivalent to $\gamma$ fitting inside an $n \times d$ box, and the number of such $\gamma$ is exactly $p_{k}(d, n)$. 
Next, when the second part in $\nu$ is 1 , we have the following. Since $\nu_{3}=1$, the condition $\sum_{i} b_{i} \alpha_{3}^{i}=\nu_{3}=1$ implies that there is a single $i$, such that $b_{i} \alpha_{3}^{i} \neq 0$, and in fact must be 1 , so $b_{i}=\alpha_{3}^{i}=1$. After renumeration, we can assume that $i=0$ (for separation purposes) with $b_{0}=\alpha_{3}^{0}=1$ and $\alpha^{0}=(n-1-r, r, 1)$ for $r=0 \ldots n-1$. For the remaining $b$ s and $\alpha$ s we have $\sum_{i} b_{i} \alpha^{i}=(L, k)-(n-1-r, r)=(L+r-n+1, k-r)$ with $b_{1}+\cdots=d-1$, and $\left|\alpha^{i}\right|=n$. This number is now, by the previous case, $\left(q^{k-r}\right) @\left(\begin{array}{c}n+d-1 \\ n\end{array}\right)_{q}$. The total number is thus

$$
c_{(L, k, 1)}=\sum_{r=0}^{n-1}\left(q^{k-r}\right) @\left(\begin{array}{c}
n+d-1 \\
n
\end{array}\right)_{q}=\left(q^{k}\right) @ \sum_{r=0}^{n-1} q^{r}\left(\begin{array}{c}
n+d-1 \\
n
\end{array}\right)_{q}=\left(q^{k}\right) @\left(\begin{array}{c}
n \\
1
\end{array}\right)_{q}\left(\begin{array}{c}
n+d-1 \\
n
\end{array}\right)_{q} .
$$

Finally, when $\nu_{3}=2$ we have the following two distinct options:

Either there is an index $i$, such that $b_{i} \alpha_{3}^{i}=\nu_{3}=2$, or $i<j$ with $b_{i} \alpha_{3}^{i}=1$ and $b_{j} \alpha_{3}^{j}=1$.

In the first case we have $b_{i} \alpha_{i}^{3}=2$ - either $b_{i}=2$, in which case $\alpha^{i}=(n-1-r, 1)$ and the rest of the $b$ 's sum to $d-b_{i}=d-2$, which brings us to the previous case (of $(L, k, 1)$ ), so the number is

$$
\left(q^{k}\right) @\left(\begin{array}{c}
n \\
1
\end{array}\right)_{q}\left(\begin{array}{c}
n+d-2 \\
n
\end{array}\right)_{q} .
$$

Otherwise, $b_{i}=1$ and $\alpha_{3}^{i}=2$. As in the case $\nu_{3}=1$, let $i=0$ and $\alpha^{0}=(n-2-r, r, 2)$, $b_{0}=1$, so we are looking for the number of $\left(b_{1}, \ldots\right)$ with $|b|=d-1$ and such that $\sum_{i} b_{i} \alpha^{i}=(L-n+r+2, k-r)$ for all possible $r=0, \ldots, n-2$. So this is

$$
\sum_{r=0}^{n-2}\left(q^{k-r}\right) @\left(\begin{array}{c}
n+d-1 \\
n
\end{array}\right)_{q}=\left(q^{k}\right) @\left(\begin{array}{c}
n-1 \\
1
\end{array}\right)_{q}\left(\begin{array}{c}
n+d-1 \\
n
\end{array}\right)_{q}
$$

Last, when there are $i<j$ with $b_{i} \alpha_{3}^{i}=1$ and $b_{j} \alpha_{3}^{j}=1$, let $i=-1, j-0$ (again, renumerating for simplicity), with $\alpha^{-1}=\left(n-1-r_{1}, r_{1}, 1\right)$ and $\alpha^{0}=\left(n-1-r_{2}, r_{2}, 1\right)$ with $0 \leq r_{1}<r_{2} \leq n-1$. We thus have for the remaining $\alpha$ and $b$ s that $b_{1}+\cdots=d-2$, and $\sum_{i} b_{i} \alpha^{i}=\left(L-\left(n-1-r_{1}\right)-\left(n-1-r_{2}\right), k-r_{1}-r_{2}\right)$. By the first case, this is

$\left(q^{k-r_{1}-r_{2}}\right) @\left(\begin{array}{c}n+d-2 \\ n\end{array}\right)_{q}$. Summing over all possible $0<r_{1}<r_{2} \leq n-1$, we have

$$
\begin{aligned}
\left(q^{k}\right) @ \sum_{0 \leq r_{1}<r_{2} \leq n-1} q^{r_{1}+r_{2}}\left(\begin{array}{c}
n+d-2 \\
n
\end{array}\right)_{q} & =\left(q^{k}\right) @ q \sum_{0 \leq r_{1} \leq r_{2}-1 \leq n-2} q^{r_{1}+\left(r_{2}-1\right)}\left(\begin{array}{c}
n+d-2 \\
n
\end{array}\right)_{q} \\
& =\left(q^{k}\right) @ q\left(\begin{array}{c}
n-2+2 \\
2
\end{array}\right)_{q}\left(\begin{array}{c}
n+d-2 \\
n
\end{array}\right)_{q},
\end{aligned}
$$

where the last identity follows from interpreting $\left(r_{2}-1, r_{1}\right)$ as a partition in the $2 \times n-2$ rectangle. Summing over all the cases considered here, we get the desired total coefficient.

- Proposition 4.2. The plethysm coefficient for $\lambda=(L, r, 2)$ is equal to

$$
\begin{aligned}
a_{\lambda}(d[n])= & \left(q^{r+1}\right) @\left(\left(\begin{array}{c}
n+d-2 \\
n
\end{array}\right)_{q} \frac{q\left(1-q^{n}\right)\left(1-q^{2}+q-q^{n}\right)}{1-q^{2}}\right. \\
& \left.+\left(\begin{array}{c}
n+d-1 \\
n
\end{array}\right)_{q}\left(q^{n+1}-1\right)+(1-q)\left(\begin{array}{c}
n+d \\
n
\end{array}\right)_{q}\right)
\end{aligned}
$$

Proof. Following equation (10), we have that $a_{\lambda}(d[n])=c_{(L, r, 2)}-c_{(L, r+1,1)}-c_{(L+1, r-1,2)}+c_{(L+1, r+1,0)}+c_{(L+2, r-1,1)}-c_{(L+2, r, 0)}$. Substituting the formulas for the $c$ 's from Proposition 4.1, and observing that $\left(q^{r+j}\right) @ f=$ $\left(q^{r}\right) @ q^{-j} f$ for any $j$, we have that

$$
\begin{array}{r}
a_{\lambda}(d[n])=\left(q^{r+1}\right) @\left(\left(q-q^{2}\right)\left(\left(\begin{array}{c}
n \\
1
\end{array}\right)_{q}\left(\begin{array}{c}
n+d-2 \\
n
\end{array}\right)_{q}+\left(\begin{array}{c}
n-1 \\
1
\end{array}\right)_{q}\left(\begin{array}{c}
n+d-1 \\
n
\end{array}\right)_{q}+q\left(\begin{array}{c}
n \\
2
\end{array}\right)_{q}\left(\begin{array}{c}
n+d-2 \\
n
\end{array}\right)_{q}\right)\right. \\
\left.+\left(q^{2}-1\right)\left(\begin{array}{c}
n \\
1
\end{array}\right)_{q}\left(\begin{array}{c}
n+d-1 \\
n
\end{array}\right)_{q}+(1-q)\left(\begin{array}{c}
n+d \\
n
\end{array}\right)_{q}\right)
\end{array}
$$

Simplifying the above expression by grouping terms for the same binomial coefficients together we obtain

$$
a_{\lambda}(d[n])=\left(q^{r+1}\right) @\left(\left(\begin{array}{c}
n+d-2 \\
n
\end{array}\right)_{q} \frac{q\left(1-q^{n}\right)\left(1-q^{2}+q-q^{n}\right)}{1-q^{2}}+\left(\begin{array}{c}
n+d-1 \\
n
\end{array}\right)_{q}\left(q^{n+1}-1\right)+(1-q)\left(\begin{array}{c}
n+d \\
n
\end{array}\right)_{q}\right) .
$$


- Proposition 4.3. Let $\lambda=(L, r, 2)$. We have that

$$
a_{\lambda}(d[n])-a_{\lambda}(n[d])=\left(q^{r}\right) @\left(\begin{array}{c}
n+d-2 \\
n-1
\end{array}\right)_{q}\left(q^{n}-q^{d}\right) \frac{\left(1-q^{d-1}\right)\left(1-q^{n-1}\right)}{\left(1-q^{d}\right)\left(1-q^{n}\right)} .
$$

Proof. Set $[a] !_{q}:=(1-q) \cdots\left(1-q^{a}\right)$, a variant of the usual factorial $q$-analogue but multiplied by $(1-q)^{a}$, and consider the desired difference via the formula in Proposition 4.2:

$$
\begin{aligned}
& a_{\lambda}(d[n])-a_{\lambda}(n[d])=\left(q^{r+1}\right) @ \\
& \left\{\left(\left(\begin{array}{c}
n+d-2 \\
n
\end{array}\right)_{q} \frac{q\left(1-q^{n}\right)\left(1-q^{2}+q-q^{n}\right)}{1-q^{2}}+\left(\begin{array}{c}
n+d-1 \\
n
\end{array}\right)_{q}\left(q^{n+1}-1\right)\right.\right. \\
& \left.-\left(\begin{array}{c}
n+d-2 \\
d
\end{array}\right)_{q} \frac{q\left(1-q^{d}\right)\left(1-q^{2}+q-q^{d}\right)}{1-q^{2}}-\left(\begin{array}{c}
n+d-1 \\
d
\end{array}\right)_{q}\left(q^{d+1}-1\right)\right) \\
& =\frac{[n+d-2] !_{q}}{[n-2] !_{q}[d-2] !_{q}} \frac{q\left(q^{n-1}-q^{d-1}\right)}{\left(1-q^{n-1}\right)\left(1-q^{d-1}\right)}-\frac{[n+d-1] !_{q}}{[n-1] !_{q}[d-1] !_{q}} \frac{(1-q)\left(q^{n}-q^{d}\right)}{\left(1-q^{n}\right)\left(1-q^{d}\right)} \\
& =\left(\begin{array}{c}
n+d-2 \\
n-1
\end{array}\right)_{q}\left(q^{n}-q^{d}\right)\left(1-\frac{\left(1-q^{n+d-1}\right)(1-q)}{\left(1-q^{n}\right)\left(1-q^{d}\right)}\right) \\
& \left.=\left(\begin{array}{c}
n+d-2 \\
n-1
\end{array}\right)_{q}\left(q^{n}-q^{d}\right) \frac{q\left(1-q^{d-1}\right)\left(1-q^{n-1}\right)}{\left(1-q^{d}\right)\left(1-q^{n}\right)}\right\}
\end{aligned}
$$

Finally, observe that the RHS is a polynomial divisible by $q$, so the coefficient at $q^{r+1}$ is the same as the coefficient at $q^{r}$ after dividing by $q$.

We are now ready to prove Theorem 3.4 as a Corollary of the above computations:

- Corollary 4.4. [Theorem 3.4] Let $d=n+1$ and $\lambda=\left(n^{2}+n-2-r, r, 2\right)$. Then $a_{\lambda}((n+1)[n])-a_{\lambda}(n[n+1]) \geq 0$, with

$$
a_{\lambda}((n+1)[n])-a_{\lambda}(n[n+1])= \begin{cases}0, & \text { when } r<n, \\ 1, & \text { when } r=n, \\ >0, & \text { when } r>n \text { and } n \geq 7,\end{cases}
$$

with the exception in the last case when $n=8$, and $r=35$ when $a_{(35,35,2)}(9[8])=$ $a_{(35,35,2)}(8[9])$.

Proof. Then by the Proposition 4.3 we have

$$
\begin{array}{r}
a_{\lambda}(n+1[n])-a_{\lambda}(n[n+1])=\left(q^{r}\right) @\left(\begin{array}{c}
2 n-1 \\
n-1
\end{array}\right)_{q}\left(q^{n}-q^{n+1}\right) \frac{\left(1-q^{n}\right)\left(1-q^{n-1}\right)}{\left(1-q^{n+1}\right)\left(1-q^{n}\right)} \\
=\left(q^{r}\right) @\left(\begin{array}{c}
2 n-1 \\
n-1
\end{array}\right)_{q}\left(q^{n}-q^{n+1}\right) \frac{\left(1-q^{n-1}\right)}{1-q^{n+1}}=\left(q^{r}\right) @\left(\begin{array}{c}
2 n-1 \\
n-2
\end{array}\right)_{q}\left(q^{n}-q^{n+1}\right)
\end{array}
$$

The last line follows by absorbing the fraction into the $q$-binomial coefficient. It is now evident, that since the $q$-binomial coefficient expands into a polynomial of $q$ (with coefficients given by $p_{*}(n-2, n+1)$ ), multiplying it with $q^{n}$ or $q^{n+1}$ gives two polynomials whose lowest order terms are $q^{n}$ and $q^{n+1}$ respectively. So if $r<n$, there is no term of such degree, and the coefficient is 0 . when $r=n$ we see that such term can only come from the first polynomial's first (lowest order) term, which is exactly $q^{n}$ since $\left(\begin{array}{c}2 n-1 \\ n-2\end{array}\right)_{q} q^{n}=q^{n}\left(1+q+2 q^{2}+\cdots\right)=$ $q^{n}+O\left(q^{n+1}\right)$. Therefore we obtain the case $r=n$.

Let now $r>n$, and set $r=n+k+1$ for some $k \geq 0$. We have that

$$
\begin{array}{r}
a_{\lambda}((n+1)[n])-a_{\lambda}(n[n+1])=\left(q^{k+1}\right) @\left(\begin{array}{c}
2 n-1 \\
n-2
\end{array}\right)_{q}-\left(q^{k}\right) @\left(\begin{array}{c}
2 n-1 \\
n-2
\end{array}\right)_{q} \\
=p_{k+1}(n+1, n-2)-p_{k}(n+1, n-2) \\
=g\left(\left(n^{2}-n-3-k, k+1\right),(n+1)^{n-2},(n+1)^{n-2}\right)>0,
\end{array}
$$


where $g$ denotes the Kronecker coefficient for the symmetric group $\mathfrak{S}_{n}$ for the 3 given partititons, and the last identity and the strict positivity are shown to hold for $n \geq 9$ in [26], and the other cases are verified by direct expansion of the $q$-binomial coefficients. In particular, we have that $p_{26}(9,6)=227=p_{27}(9,6)$ which gives the only exceptional 0 plethysm.

\section{Computer calculations}

- Proposition 5.1. If $X$ is defined as in Proposition 3.6, then for all $\mu \in X$ of length 3 we have $\operatorname{mult}_{\mu}\left(\mathbb{C}\left[\mathrm{Ch}_{3}^{6}\right]\right)>0$.

If $X$ is the set of generators of the semigroup of 4-partitions $\mu$ that have $a_{\mu}(d[7])>0$ (see full version of this paper), then for all $\mu \in X$ we have mult ${ }_{\mu}\left(\mathbb{C}\left[\mathrm{Ch}_{4}^{7}\right]\right)>0$.

Proof. Proposition 5.1 is proved by a computer calculation that is a refinement and speedup of the computation performed in [9]. Indeed, a run of the method from [9] would take significantly too long to prove Proposition 5.1 in any reasonable time. Our new method makes extensive use of memory resources, while the method from [9] uses almost no memory. The description of the computation can be found in the full version of this paper.

\section{References}

1 Markus Bläser and Christian Ikenmeyer. Introduction to geometric complexity theory. lecture notes, summer 2017 at Saarland University, version July 25, 2018, http://people.mpi-inf . mpg.de/ cikenmey/teaching/summer17/introtogct/gct.pdf, 2018.

2 Emmanuel Briand, Rosa Orellana, and Mercedes Rosas. Reduced Kronecker coefficients and counter-examples to Mulmuley's strong saturation conjecture SH. Computational Complexity, 18:577-600, 2009.

3 Peter Bürgisser, Michael Clausen, and M. Amin Shokrollahi. Algebraic complexity theory, volume 315 of Grundlehren der Mathematischen Wissenschaften [Fundamental Principles of Mathematical Sciences]. Springer-Verlag, Berlin, 1997. With the collaboration of Thomas Lickteig.

4 Peter Bürgisser, Jesko Hüttenhain, and Christian Ikenmeyer. Permanent versus determinant: not via saturations. Proceedings of the American Mathematical Society, 145(3):1247-1258, 2017.

5 Peter Bürgisser and Christian Ikenmeyer. The complexity of computing Kronecker coefficients. In FPSAC 2008, Valparaiso-Viña del Mar, Chile, DMTCS proc. AJ, pages 357-368, 2008.

6 Peter Bürgisser and Christian Ikenmeyer. Geometric Complexity Theory and Tensor Rank. Proceedings 43rd Annual ACM Symposium on Theory of Computing 2011, pages 509-518, 2011.

7 Peter Bürgisser and Christian Ikenmeyer. Explicit Lower Bounds via Geometric Complexity Theory. Proceedings 45th Annual ACM Symposium on Theory of Computing 2013, pages 141-150, 2013.

8 Peter Bürgisser, Christian Ikenmeyer, and Greta Panova. No occurrence obstructions in geometric complexity theory. Journal of the AMS, 32:163-193, 2019. An earlier version was presented at the IEEE 57th Annual Symposium on Foundations of Computer Science (FOCS) 2016 in New Brunswick, New Jersey.

9 Man-Wai Cheung, Christian Ikenmeyer, and Sevak Mkrtchyan. Symmetrizing tableaux and the 5th case of the Foulkes conjecture. Journal of Symbolic Computation, 80(3):833-843, 2016. doi:10.1016/j.jsc.2016.09.002.

10 W. Fulton. Young tableaux, volume 35 of London Mathematical Society Student Texts. Cambridge University Press, Cambridge, 1997.

11 Roe Goodman and Nolan R. Wallach. Symmetry, representations, and invariants, volume 255 of Graduate Texts in Mathematics. Springer, Dordrecht, 2009. 
12 Yonghui Guan. Brill's equations as a GL(V)-module. Linear Algebra and its Applications, 548:273-292, 2018. doi:10.1016/j.laa.2018.02.026.

13 Charles Hermite. Sur la théorie des fonctions homogenes à deux indéterminées. Cambridge and Dublin Mathematical Journal, 9:172-217, 1854.

14 Christian Ikenmeyer. Geometric Complexity Theory, Tensor Rank, and Littlewood-Richardson Coefficients. PhD thesis, Institute of Mathematics, University of Paderborn, 2012. URL: http://nbn-resolving.de/urn:nbn:de:hbz:466:2-10472.

15 Christian Ikenmeyer. GCT and symmetries. unpublished lecture notes, version from January 29, 2018.

16 Christian Ikenmeyer and Greta Panova. Rectangular Kronecker Coefficients and Plethysms in Geometric Complexity Theory. Advances in Mathematics, 319:40-66, 2017. An earlier version was presented at the IEEE 57th Annual Symposium on Foundations of Computer Science (FOCS) 2016 in New Brunswick, New Jersey.

17 Harlan Kadish and J. M. Landsberg. Padded Polynomials, Their Cousins, and Geometric Complexity Theory. Communications in Algebra, 42(5):2171-2180, 2014. doi:10.1080/ 00927872.2012 .758268 .

18 Hanspeter Kraft. Geometrische Methoden in der Invariantentheorie. Friedr. Vieweg und Sohn Verlagsgesellschaft, Braunschweig, 1985.

19 J. M. Landsberg. Geometry and Complexity Theory. Cambridge Studies in Advanced Mathematics. Cambridge University Press, 2017. doi:10.1017/9781108183192.

20 Joseph Landsberg. Tensors: Geometry and Applications, volume 128 of Graduate Studies in Mathematics. American Mathematical Society, Providence, Rhode Island, 2011.

21 I. G. Macdonald. Symmetric functions and Hall polynomials. Oxford Mathematical Monographs. The Clarendon Press, Oxford University Press, New York, second edition, 1995. With contributions by A. Zelevinsky, Oxford Science Publications.

22 K.D. Mulmuley and M. Sohoni. Geometric Complexity Theory. I. An approach to the P vs. NP and related problems. SIAM J. Comput., 31(2):496-526 (electronic), 2001.

23 K.D. Mulmuley and M. Sohoni. Geometric Complexity Theory. II. Towards explicit obstructions for embeddings among class varieties. SIAM J. Comput., 38(3):1175-1206, 2008.

24 D. Mumford. Algebraic geometry. I: Complex projective varieties. Classics in mathematics. Springer-Verlag, Berlin, 1995. Reprint of the 1976 edition in Grundlehren der mathematischen Wissenschaften, vol. 221.

25 Hariharan Narayanan. On the complexity of computing Kostka numbers and LittlewoodRichardson coefficients. J. Algebraic Combin., 24(3):347-354, 2006.

26 Igor Pak and Greta Panova. Strict unimodality of $q$-binomial coefficients. C. R. Math. Acad. Sci. Paris, 351(11-12):415-418, 2013. doi:10.1016/j.crma.2013.06.008.

27 Claudio Procesi. Lie groups. Universitext. Springer, New York, 2007. An approach through invariants and representations.

28 Ramprasad Saptharishi. A survey of lower bounds in arithmetic circuit complexity. , version 3.1.4, 2017. URL: https://github.com/dasarpmar/lowerbounds-survey.

29 Richard P. Stanley. Enumerative combinatorics. Vol. 2, volume 62 of Cambridge Studies in Advanced Mathematics. Cambridge University Press, Cambridge, 1999. With a foreword by Gian-Carlo Rota and appendix 1 by Sergey Fomin.

30 Richard P. Stanley. Positivity problems and conjectures in algebraic combinatorics. In Mathematics: frontiers and perspectives, pages 295-319. Amer. Math. Soc., Providence, RI, 2000.

31 Richard P. Stanley. Enumerative combinatorics. Vol. 1. Cambridge University Press, Cambridge, 2011. second edition.

32 Bernd Sturmfels. Algorithms in Invariant Theory. Texts \& Monographs in Symbolic Computation. Springer, 2008. 